\title{
Revising Cataloging Rules and Standards to Meet the Needs of People with Disabilities
}

\section{A Proposal for South Korea}

\author{
Seungmin Lee, Taewoo Nam, and Youngjoon Nam
}

Various types of alternative format materials have been developed and acquired by libraries to improve information accessibility for people with disabilities. Although many cataloging standards contain specific rules designed to describe unique characteristics of these materials, most of these rules focus on describing braille books. This lack of alternative format-specific cataloging rules may hinder information accessibility for people with disabilities. This paper proposes revision, modification, and expansion of the Korean Cataloging Rules, 4th ed., and to Korean MARC (KORMARC) fields to address these problems and to comprehensively describe various types of alternative format materials. Although these changes are intended for the library community in South Korea, they may be relevant to other countries.

Seungmin Lee (ableman@cnu.ac.kr) is Assistant Professor, Chungnam National University, Daejeon, South Korea; Taewoo Nam (namtw@cau.ac.kr) is Professor, and Youngjoon Nam (namyj@cau. ac.kr) is Professor, Chung-Ang University, Seoul, South Korea.

Submitted April 9, 2012; tentatively accepted for publication pending revision on May 17, 2012; revision submitted June 14, 2012, and accepted for publication June 30, 2012.
S ince the enactment of the Americans with Disabilities Act (ADA) in 1990 in

$\mathcal{S}$ the United States, interest in information accessibility for people with disabilities has increased in the library community. ${ }^{1}$ A survey found that many libraries tried to assist people with different kinds of disabilities by eliminating physical barriers to facilities and by retrieving materials from stacks. ${ }^{2}$ In addition to these improvements, libraries have continued to acquire alternative format materials, including talking books, video with subtitles or sign language, braille books, and other materials, to satisfy the information needs of individuals with disabilities. ${ }^{3}$ With the development of information technologies, new types of alternative format materials have been produced, and traditional alternative formats materials, such as braille books, have increased.

Format-specific cataloging rules have become necessary because these alternative format materials have unique characteristics. Although many cataloging standards already contain rules designed to describe those materials, most focus on describing one specific type-braille books. In addition, these rules may not be sufficient to describe the unique characteristics of braille materials because they are based on rules designed for general materials, especially the printed book format. Furthermore, new types of alternative formats may have characteristics different from braille books as well as from as general materials. Current cataloging rules may not fully support the detailed description of these alternative formats and hinder information accessibility for people with disabilities. 
The library community in South Korea faces the same problems encountered in English-speaking countries when trying to appropriately describe alternative formats for people with disabilities. In South Korea, the Korean Cataloging Rules (KCR) have been a national standard since 1964 when the first edition was released. ${ }^{4}$ Korean Cataloging Rules, 3rd ed. (KCR3), released in 1983, was revised so that most rules follow international cataloging rules. ${ }^{5} \mathrm{KCR}$ was influenced by International Standard Bibliographic Description (ISBD) and the Anglo-American Cataloguing Rules. ${ }^{6}$ KCR4, the fourth and current edition of the cataloging rules, has been adopted by most libraries in South Korea as the standard. ${ }^{7}$ Korean Machine Readable Cataloging (KORMARC), influenced by USMARC, provides a structure for the records in a machine-readable format that is based on the rules in the KCRs. Most of the bibliographic records in South Korea are now in KORMARC format. ${ }^{8}$

KCR4 and KORMARC are designed to be adopted by all libraries in South Korea, including public, university, school libraries, and libraries for people with disabilities. However, because of the lack of detailed rules for alternative format materials, many libraries cannot fully describe those materials in the appropriate manner. Even more problematic, some libraries have altered the rules to satisfy their own purpose or for their convenience in describing those materials. These problems have resulted in inconsistent, isolated, and noninteroperable records for the same alternative format materials.

To understand the extent of the problems, the authors analyzed a set of 25,284 catalog records for alternative format materials. To overcome the problems outlined above, the authors proposes a specific set of rules for KCR4 and KORMARC fields to comprehensively describe various types of alternative format materials and to reflect their unique characteristics. The authors do not create completely new cataloging rules, but propose revision, modification, and expansion of existing rules. These changes can be applied to the library community in South Korea and may have value to the larger cataloging community.

\section{People with Disabilities and Alternative Format Materials to Meet Their Needs}

No single definition of "disability" exists because of its many possible characteristics. Todaro defined "disability" as a physical or mental condition that partially or completely prohibits an individual from the use of his or her body to perform daily tasks. ${ }^{9}$ Another commonly cited definition comes from the World Health Organization: "Disability is an umbrella term, covering impairments, activity limitations, and participation restrictions." ${ }^{10}$ The International Federation of Library Associations and Institutions (IFLA) specifically defines print disability as the inability to read standard print or the inability to physically hold a book. ${ }^{11}$

In South Korea, disability refers to physical or mental impairment or dysfunction that severely affects and constrains social life of an individual. ${ }^{12}$ Based on this definition, the legislation of South Korea defines people with disabilities as individuals with difficulties in using facilities, moving, or accessing information caused by physical or mental impairments, whether such impairments are long-term or recurring. ${ }^{13}$

Based on these definitions, the range of disabilities and people with disabilities is quite broad-from physical impairment to inability to access information. Print disability substantially limits the individual in seeing or reading traditional print materials in its original form. To improve information accessibility of people with disabilities, the library communities in many countries have increased provision of alternative format materials. For example, the Library of Congress National Library Services for the Blind and Physically Handicapped (NLS) describes various formats that have been developed and used to support people with disabilities, including braille books, audiobooks, large print, and digital books that can be accessed via voice software. ${ }^{14}$

The Committee of Libraries Serving Disadvantaged Persons in IFLA suggests media formats that libraries should acquire to serve people with disabilities as follows: talking books, talking newspapers, talking periodicals, largeprint books, easy-to-read books, braille books, video books with subtitles or sign language, e-books, and tactile picture books. ${ }^{15}$ The publication of these alternative format materials has continued to increase, and new technologies have supported the development of various alternative formats. South Korea also has tried to develop and publish more alternative formats to improve information accessibility for people with disabilities. Through government financial support, the Korean Library Association (KLA) has implemented a plan to increase the percent of alternative format materials in library collections from 2 percent in 2009 to 10 percent by 2013. The funds required to proceed with this plan are increasing from around $\$ 800,000$ in 2009 to around $\$ 2$ million in $2013 .{ }^{16}$

\section{Cataloging Rules for Alternative Format Materials}

The increasing number of alternative format materials being published has likewise increased the need for tools to fully describe the unique characteristics of those materials. To satisfy these needs, current cataloging codes contain specific rules that can be applied to alternative format materials.

\section{Anglo-American Cataloguing Rules}

The Anglo-American Cataloguing Rules, 2nd rev. ed. 
Table 1. AACR2R Lists of General Material Designation

\begin{tabular}{|c|c|c|}
\hline List 1 (Britain) & $\begin{array}{l}\text { List } 2 \text { (Australia, Ca } \\
\text { and the United Stat }\end{array}$ & $\begin{array}{l}\text { ada, } \\
\text { s) }\end{array}$ \\
\hline \multirow{2}{*}{$\begin{array}{l}\text { Braille } \\
\text { Cartographic mate- } \\
\text { rials }\end{array}$} & Activity card & Microscope slide \\
\hline & $\begin{array}{l}\text { Art original } \\
\text { Art reproduction }\end{array}$ & $\begin{array}{l}\text { Model } \\
\text { Motion picture }\end{array}$ \\
\hline Electronic resource & Braille & Music \\
\hline Graphic & Cartographic material & Picture \\
\hline Manuscript & Chart & Realia \\
\hline Microform & Diorama & Slide \\
\hline Motion picture & Electronic resource & Sound recording \\
\hline Multimedia & Film strip & Technical drawing \\
\hline Music & Flash card & Text \\
\hline Object & Game & Transparency \\
\hline Sound recording & Kit & Toy \\
\hline Text & Manuscript & Video recording \\
\hline Video recording & Microform & \\
\hline
\end{tabular}

(AACR2R) provides two types of General Material Designations (GMD): List 1 and List $2 .{ }^{17}$ List 1 is used in Britain and contains thirteen types of resources; List 2 is for Australia, Canada, and the United States, and contains twenty-seven resource types (see table 1). Both lists contain braille as a resource type. The description part of AACR2R, however, only provides cataloging rules for eleven resource types (see table 2).

As shown in table 2, general rules for description do not include any alternative format, although GMD provides braille as a resource type. Instead, $A A C R 2 R$ provides description rules stipulating that the description of alternative format materials should follow the rules for other types of resources with the addition of "(large print $)$ " or "(tactile)" for the visually impaired. ${ }^{18}$ General rules for sound recordings may be applied to describe audio materials. However, the rules for sound recordings may not fully describe alternative format materials, even audio format, because those materials have characteristics different from the audio materials for general users.

\section{Korean Cataloging Rules}

The structure of KCR4 is similar to AACR2R because $K C R$ are based on the Anglo-American Cataloguing Rules and have adopted most of these rules since the code's origin . Similar to AACR2R, KCR4 also provides GMD consisting of eleven categories (see table 3). ${ }^{19}$

The resource types in KCR4 are similar to those of AACR2R. However, one major difference between the two is that KCR4 provides description rules for alternative format materials, which are commonly named "braille book." The type "braille books" includes a broad range of alternative format materials, including cartographic materials, printed music scores, and continuing resources in tactile format. The description rules may cover the complete characteristics of each material, including title proper, statement
Table 2. Resource Types in AACR2R

\begin{tabular}{|c|c|}
\hline $\begin{array}{l}\text { Books, pamphlets, and printed } \\
\text { sheets } \\
\text { Cartographic materials } \\
\text { Manuscripts } \\
\text { Music } \\
\text { Sound recordings } \\
\text { Motion pictures and video record- } \\
\text { ings }\end{array}$ & $\begin{array}{l}\text { Graphic materials } \\
\text { Electronic resources } \\
\text { Three-dimensional artifacts and } \\
\text { realia } \\
\text { Microforms } \\
\text { Continuing resources }\end{array}$ \\
\hline Table 3. Resource Types in KCR & \\
\hline $\begin{array}{l}\text { Monograph } \\
\text { Cartographic materials } \\
\text { Antique books } \\
\text { Printed music scores } \\
\text { Sound recordings } \\
\text { Motion pictures and video record- } \\
\text { ings }\end{array}$ & $\begin{array}{l}\text { Electronic resources } \\
\text { Three-dimensional artifacts and } \\
\text { realia } \\
\text { Microforms } \\
\text { Continuing resources } \\
\text { Braille books }\end{array}$ \\
\hline
\end{tabular}

of responsibility, edition, publication and distribution, physical description, series, and note area.

Although KCR4 provides alternative format-specific description rules, many problems have arisen when applying the rules to create catalog records. The rules mainly focus on describing braille books because the KCR4 prescribes the rules as "description for braille materials." Other types of alternative formats are rarely mentioned. Another problem is applying existing rules for general materials to alternative format materials. Most description rules for alternative formats in KCR4 are quoted from the rules for general materials with few additional clauses that can be applied to alternative formats.

The authors searched the literature for publications specifically addressing cataloging of alternative formats for people with disabilities and was unable to find any.

\section{Data Collection and Method of Analysis}

This research analyzed bibliographic records for alternative format materials currently used in the library community in South Korea to identify the inconsistencies and inaccuracies in those records. Most records came from the General Catalog for Format Materials for the Blind, which contains approximately 100,000 bibliographic records for alternative format materials contributed by public libraries, university libraries, and libraries for people with disabilities in an effort to standardize cataloging records for those materials. Another data set came from Korea Library Automation System in Able (KOLASIA), which is a major part of the Database of National Cataloging for Alternative Format Materials of South Korea. The National Library of Korea has been constructing this database since 2010. Twelve libraries for people with disabilities participate by providing 
their cataloging records for alternative format materials. KOLASIA, which is designed to provide one-stop services for those materials, currently contains about 120,000 records for alternative format materials and has been adopted as a national database for bibliographic records for those materials. Among these records, the authors examined a subset that consisted of two or more parallel records for the same item.

The data set that the authors analyzed consisted of bibliographic records for several types of alternative format materials: braille books in printed format $(12,748)$, braille books in electronic format $(2,717)$, audiobooks $(9,213)$, picture books with tactile label (586), and video resources (20). All 25,284 records are in the KORMARC format. The authors examined each KORMARC field in parallel records and compared the values with each other. During this analysis process, each record was entered into a spreadsheet divided into each KORMARC field. The same field with different values was identified so that unique types of resource description could be derived. Through this analysis and comparison, the authors identified different patterns of assigning values for a field and inconsistencies in using KORMARC fields to describe alternative format materials across libraries. Based on the findings, the authors developed revisions, modifications, and expansions of KORMARC fields to fully describe alternative format materials.

\section{Findings and Analysis Fields Related to Alternative Format Materials in KORMARC}

KORMARC contains numerous fields to describe various types of materials, from monographs to electronic resources. Although some of these fields are not alternative formatspecific, they are currently used in describing alternative format materials. The fields that are used in describing alternative format materials are shown in table 4 .

Among these fields, the most frequently used are 245 (Title Statement) \$h (Medium), 260 (Publication, Distribution, etc.), 300 (Physical Description), 500 (General Note), 700 (Added Entry-Personal Name), and 710 (Added Entry-Corporate Name). Only the 260 and 300 fields appeared in all records. However, some of the values of these fields are not from alternative format materials but from the original materials. In addition, some fields, such as the 521 field (Target Audience Note), are rarely used even though they may be essential to describe alternative format materials. These inconsistent and inappropriate uses of fields may result in incomplete records and cause difficulties in retrieving alternative format materials.

Several other fields have been used, including 650 (Subject Added Entry-Topical Term), 653 (Index
Term-Uncontrolled), and 655 (Index Term-Genre/ Form), to describe the subject or genre/form of alternative format materials. However, they are not alternative formatspecific. For this reason, the author did not consider those fields during analysis.

\section{Primary Source for Description}

The content of the alternative format materials may be an abridged or reduced version of the original material. Although they share the same or similar content, these two formats should be cataloged as different items because they are published for different users and in different mediums.

The description rules in KCR4 do not provide any specific statements about how to differentiate these formats. As a result, different libraries have distorted the rules to satisfy their own purposes or for their convenience in creating records for alternative format materials. For example, most libraries create records from the perspective of the original material even if the record is for an alternative format. In this case, the description of the alternative formats usually has been put in a note area or added using inappropriate fields. This frequently happened when creating a record for items in sound recording format, such as MP3 or Digital Accessible Information System (DAISY) files. Although these formats can be described in a computer file or data note area, the records in the data sets put the description in the general note area with simple introductory terms. Examination of the 9,213 records for audiobooks in the data set found 2,041 records that use of the general note area for this description. This makes the record ambiguous because identifying whether it is describing the original material or an alternative format is difficult.

\section{Person (or Institution) Participating in the Publication of Alternative Format Materials}

According to current cataloging rules for alternative format materials, the author of the original material is described in the 245 field with subfield code $\$ \mathrm{~d}$ (Statement of Responsibility). This is reasonable because the author of the original material is responsible for the content of the material even if the record is for an alternative format. The individuals who participated in publishing alternative format materials, such as the reader or narrator, are only described in the 511 field (Participant or Performer Note), which was originally designed to describe the participant or performer who contributed to creating the content. In this case, making the reader or narrator an "added entry" in the 700 field (Added Entry-Personal Name) or 710 field (Added Entry-Corporate Name) as well as in the 511 field would be better because it would provide additional access points. In most records, however, the 700 and 710 fields are rarely 
Table 4. KORMARC Fields Used to Describe Alternative Format Materials

\begin{tabular}{|c|c|c|c|}
\hline \multirow[b]{2}{*}{ Field } & \multirow[b]{2}{*}{ Subfield Code } & \multicolumn{2}{|c|}{ Occurrence } \\
\hline & & No. & $\%$ \\
\hline \multirow[t]{2}{*}{245 - Title Statement } & $\$ \mathrm{~d}-$ Statement of responsibility & 25,284 & 100.0 \\
\hline & $\$ n-$ Number of part/section of a work & 15,251 & 60.3 \\
\hline 260 - Publication, Distribution, etc. & & 25,284 & 100.0 \\
\hline 500 - General Note & $\$ \mathrm{a}-$ General note & 18,807 & 74.4 \\
\hline 511 - Participant or Performer Note & $\$ \mathrm{a}-$ Participant or performer note & 8 & 0.0 \\
\hline 518 - Date/Time and Place of an Event Note & & 404 & 1.6 \\
\hline 521 - Target Audience Note & $\$ \mathrm{a}-$ Target audience note & 16,436 & 65.0 \\
\hline 534 - Original Version Note & & 67 & 0.3 \\
\hline
\end{tabular}

used. Even more problematic, most records leave the 511 field blank. Only 8 of the 25,284 records in the data set used the 511 field. Instead, the 500 field was occasionally used to describe these individuals using plain text.

KORMARC stipulates using the 500 field to describe detailed information about the original material and braille translator. Not quite one-fourth $(2,999$ of 12,748$)$ of the records for braille books in the data set describe the braille translator in this field. However, the description about braille translator may not be considered as general information appropriate for the 500 field and would be better described using another field.

\section{Types of Alternative Format Materials}

Subfield code $\$$ h in the 245 field is used to describe various types of alternative format materials. Similar to MARC, KORMARC indicates these materials using brackets (e.g., [braille material]). Although various alternative formats are currently published, existing records in the data set indicate only two types: "Braille material" and "Sound recordings." This may be because KCR4 focuses on visually impaired people. The 300 field is often used to describe detailed types of materials, such as printed music score, cartographic materials, and tactile books, instead of the 245 field. In the records in the data set, however, subfield codes $\$$ a (Extent) and $\$ \mathrm{~b}$ (Other Physical Details) in the 300 field are limited to indicate the medium of alternative format materials, such as book, compact disc, cassette tape, and map, without mentioning it is an alternative format (e.g., press braille book). In addition, KCR4 does not provide a standardized way of using terms to describe the medium of the materials. For example, the medium of one sound recording is described inconsistently using the terms "Compact Disc," "CD," "Disc," or "Record."

\section{Alternative Format Materials Published in Divided Parts}

Describing alternative format materials published in several parts is one of the most prevalent problems seen in records in the data set. In many cases, especially with braille books, one original physical item is divided into several sequencing parts when it is transformed into an alternative format. In this case, the 300 field indicates the total number of the divided parts. For example, when one original physical item is transformed into four physical items in braille format, the 300 field indicates the total number using subfield code $\$$ a with the value "4 volumes." The number or name of each part is described in the 500 field rather than using the 245 field with subfield codes $\$ \mathrm{n}$ (Number of part/section of a work) and \$p (Name of part/section of a work). In addition, the 245 field cannot be used for alternative format materials because existing records have been created from the perspective of original material.

\section{Physical Description of Alternative Format Materials}

In the records in the data set, physical description of alternative format materials is currently described in the 300 field with subfield code $\$ c$ (Dimensions). It is sometimes indicated in the 500 field instead $(5,211$ of 25,284 records) One of the main reasons for this inconsistency is reliance on the catalog record for the original format. Records are created from the perspective of original material, and the 300 field is used to describe the physical description of the original material. Another reason is that KCR4 stipulates that the description part does not use the physical description area 
when describing alternative formats. However, most of the records in the data set describe the physical description of those materials using either the 300 or 500 field.

\section{Publication of Alternative Format Materials}

Another serious inconsistency can develop when using the 260 field to describe the publisher of alternative format materials. This is mainly because KCR4 has no clear rules about how to differentiate the publisher of the alternative format from the publisher of the original material. Thus the 260 field often is used to describe both publishers (found in 4,419 records or 17.5 percent of the data set). In other cases, only the publisher of the original material is given in the 260 field (found in 7,594 records or 30.0 percent of the data set). The publisher of the alternative format is not indicated in the record or occasionally is described in the 500 field (3,854 or 15.2 percent of the data set), which may not be appropriate for the publisher of alternative format materials.

\section{Target Audience of Alternative Format Materials}

The 521 field can be used to describe the specific audience or intellectual level of alternative format material. In describing alternative format materials in KORMARC, however, this field mostly is used to indicate the type of disability for which those materials are designed or published $(16,436$ or 65.0 percent of records in the data set). It is seldom used to indicate with any specificity the user groups or intellectual level of audience. Although this field is used in 14,360 (56.8 percent) of records in the data set to describe a target audience, most of these set the value of this field as "visually impaired," which is almost meaningless in indicating the target audience of alternative format materials.

\section{Original Materials of Alternative Format Materials}

Most alternative format materials are published by transforming or reproducing original material in different formats; therefore indicating the relationship between original and alternative format materials may be necessary. The 534 field is used to provide information about the original material. Although this field is expected to be used in KORMARC, KCR4 stipulates that original material should be described in the 500 field in plain text. Another problem is that records for alternative format materials are created from the perspective of original materials and add descriptions of the alternative format materials using other fields. Because of this practice, only 67 records in the data set used the 534 field, and the description of original and alternative format materials are mixed in one record.

\section{File Format of Alternative Format Materials}

Currently, various file formats are used to publish alternative format materials, especially for sound recordings. However, many catalog records in the audiobooks subset provide a simple statement in the 500 field, such as "This material is in DAISY format" or "This material is in digital format" (found in 1,162 records or 4.6 percent of the data set). No other field is used to indicate a specific file format or any system requirements. However, this information is important for individuals using these materials, especially if the digital or electronic format requires specialized equipment.

\section{Equipment Required to Use Alternative Format Materials}

Some types of alternative format materials, especially in digital or electronic format, require specific equipment or software, such as a barcode scanner, screen reader software, or text-to-speech software, to use the material. Thus indicating required equipment and software in the material description is desirable. In KORMARC, however, no field is provided for this information. Although the 500 field can be used to indicate the required equipment, no records used this field to indicate required equipment or software.

\section{Modification of KCR4 and KORMARC for Alternative Format Materials}

The authors proposed revisions and modifications to KOMARC and KCR4 to address the problems identified above. After the proposal, the authors discussed the proposed changes in three consultation meetings to prevent a theoretical bias. The consultation meeting participants consisted of four experts in cataloging rules (professors in library and information science), nine librarians in different libraries with experience serving people with disabilities, and ten individuals representing various user groups. The results of the consultation meetings were applied to the proposal and are represented in the proposed changes below.

The proposed changes are organized in three areas: revision of general cataloging rules in KCR4, modification of existing fields and subfield codes in KORMARC, and addition of new fields and subfield codes in KORMARC.

\section{Revision of General Cataloging Rules in KCR4}

\section{Primary Source for Description}

In the data set, the descriptions of both original and alternative format materials are mixed in one record, which makes determining if the record is for the original or the alternative format materials difficult. To solve this problem, the author 
Table 5. Expanded List of KCR4 Types of Alternative-Format Materials

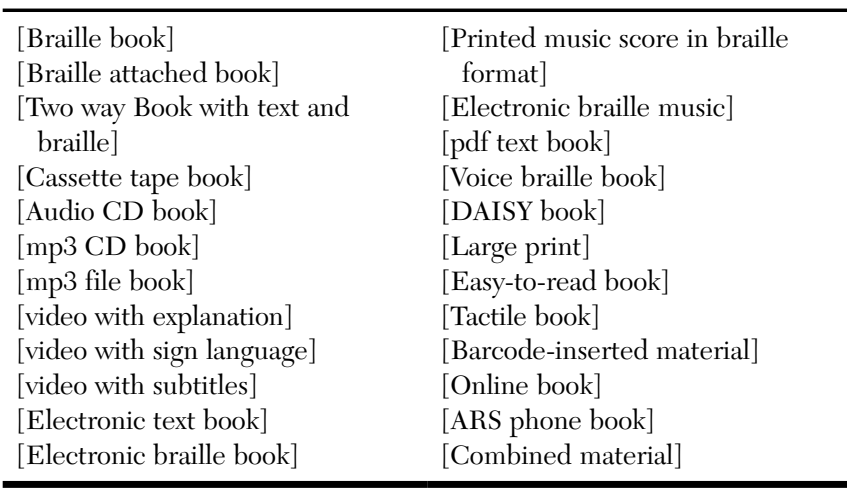

proposes creating records from the perspective of alternative format materials. A description of the original material can be added in a note area and coded in the appropriate KORMARC field. By changing the source of catalog record information from the original to the alternative format material, the details of alternative format materials can be described clearly using various fields and subfield codes without any inconsistency.

\section{Types of Alternative Format Materials}

In existing records based on KCR4, the description of alternative format materials is limited to "Braille materials" or "Sound recordings." However, many more types of alternative format materials exist. Because each type has unique characteristics and may need different equipment or software to use the material, specifying the type of various alternative format is important. Thus the authors suggest extending the types of alternative formats provided by KCR4. Table 5 shows twenty-four alternative formats. This set of options covers all alternative format materials currently published in South Korea. However, new types of alternative format may be developed and published. In this case, the new types may need to be added to the list.

\section{Modification of Existing Fields and Subfield Codes in KORMARC}

\section{Leader/06-Type of Record}

In the current records, all types of alternative format materials are categorized under the type "A-Language Material." In this case, the description of alternative formats should follow the rules for general text materials, which may cause many problems in describing unique characteristics of those materials. Another ambiguity occurs when describing printed music score in braille format. This format of materials is inconsistently categorized under the type " $\mathrm{C}$ - Notated
Table 6. Extended Types of KCR4 Alternative-Format Materials in Leader/06

\begin{tabular}{ll}
\hline A - language material & I - nonmusical sound recording \\
B - alternative format materials & $\mathrm{J}$ - musical sound recording \\
C - notated music & $\mathrm{M}$ - electronic material \\
$\mathrm{D}$ - manuscript notated music & $\mathrm{O}$ - kit \\
$\mathrm{E}$ - cartographic material & $\mathrm{P}$ - mixed material \\
$\mathrm{F}$ - manuscript cartographic & $\mathrm{R}$ - three-dimensional artifact \\
$\quad$ material & (naturally occurring object) \\
G - projected medium & $\mathrm{T}$ - manuscript language material \\
K - two-dimensional non-project- & $\mathrm{W}$ - antique material \\
able graphic &
\end{tabular}

Music" or "A-Language Material" in current records. To solve this ambiguity and inconsistency, the authors propose adding a new type for alternative format materials in Leader/06: "B-Alternative format materials" (see table 6). This new type will clearly identify that the material is for people with disabilities because it indicates what type of material the rest of the record is describing.

\section{5-Title Statement (\$\$h Medium)}

In existing records, the 245 field with subfield code $\$ \mathrm{~h}$ is used to indicate the specific type of alternative format material using brackets. However, as mentioned above, various types of alternative format materials are described under only two types: "Braille materials" and "Sound recordings." Therefore indicating the specific types of the materials in material description is necessary. The authors suggest expanding the types of alternative format materials from two to twenty-three based on the modification of Leader/06 proposed in this paper (see table 6).

Although these twenty-three types may be sufficient to indicate the types of alternative format materials, some materials may not be described using any of these because they are a combination of multiple formats. For example, some braille books contain barcodes to provide text-tospeech functionality by combining braille and the barcode. This type of material could not fall into any of the specific types proposed. To indicate these multitype materials, "Combined material" is added as one of the types of alternative format materials.

\section{5-Title Statement (\$n Number of Part/Section of a Work)}

The subfield code $\$ \mathrm{n}$ in the 245 field in existing records is rarely used for alternative format materials. It is used mostly to describe the number of parts of the original material even if the record is created for an alternative format material. To indicate the sequencing parts of alternative format materials, the author proposes utilizing this field with subfield code $\$ \mathrm{n}$ 
to describe both the total number of parts and the specific number of the parts, following the form "total number of parts of original material (the part number of original material- the part number of alternative format material that is described in the record)." Following is an example of the use of the field 245 with subfield code $\$$ :

245 \#\# \$a title of a resource $\$$ h [braille book] \$n 2(1-3)

If the original material consists of two parts and transformed alternative format material is divided into seven sequencing parts (e.g., part 1 of original material is divided into three parts of alternative format and part 2 of original material into four parts of alternative format materials) and the record describes part 1 of original materials and part 3 of alternative format material, the value of the subfield code $\$ \mathrm{n}$ would be "2(1-3)." If the original material consists of one volume and alternative format material also consists of one volume, this field can be left blank. This proposed way of using the subfield code $\$ \mathrm{n}$ allows for material description to identify the sequencing parts of both original and alternative format material. In addition, the value of the subfield code $\$ \mathrm{n}$ can indicate the record is for alternative format material because its input format is different from that of original material.

\section{0-Edition statement}

If an alternative format material is revised or a new edition is published, the 250 field can be used to indicate the edition information. This field should be used only for the edition information of the alternative format material if the record is for an alternative format. The edition information for the original material can be indicated using the 534 field (Original Version Note). By separating the edition information, the retrieval of a specific edition of an alternative format material can be improved.

\section{0-Publication, Distribution, etc., Statement}

The information about publication or distribution of alternative format materials can be described using the 260 field. As with the 250 field, this field should be only for information about the alternative format material if the record is for an alternative format. The publication information of the original material should be described in the 534 field. This separation of the publication information of alternative format materials and original materials can support the consistency of the records when describing alternative format materials.

\section{0-Physical Description}

The use of the physical description field should be restricted to the alternative format material being described. Subfield code $\$$ a should be used to indicate the extent of the material followed by the number of physical pages, volumes, total duration, etc., enclosed by parenthesis. The physical description of the original material can be recorded in the 534 field or the 500 field if needed to clarify the record is for an alternative format.

\section{0-General Note}

The 500 field in KORMARC is considered a universal field that can describe a broad range of information related to alternative format material in plain text. Although KCR4 recommends this field be used to describe the braille translator or information about the original materials, it is mostly used to indicate the total page number of braille books, recording format of sound recording materials, and medium of alternative format materials. To utilize other fields and to precisely describe specific aspects of alternative format materials, the author proposes that a note for original material be assigned to the 534 field (Original Version Note). Description of the braille translator is to be placed in the 509 field, which will be discussed below. By assigning values to appropriate existing fields, the 500 field can be reserved for general descriptions of alternative format materials.

\section{6-Restrictions on Access Note}

The restrictions on access note field is rarely used in existing records. However, indicating restrictions imposed on access to an alternative format material, especially in cases where access is limited to registered patrons with disabilities, is desirable. Thus the author proposes applying this field to all records that describe alternative format materials.

\section{1-Target Audience Note (First Indicator)}

The 521 field also is rarely used in describing the target audience of alternative format materials. It is used instead for the types of disability, such as "visually impaired." However, it is necessary to describe the specific audience or intellectual level of the content even if the record is for alternative formats. Thus the author proposes using this field in the same manner as it is used for general materials, such as "For grades 3-6." The author also suggests that the first indicator should be mandatory and its value be " 3 - Special audience characteristics" to indicate that the record is for an alternative format material.

\section{0-Additional Physical Form Available Note}

If one original material is transformed into several different 
alternative formats, they are all related to each other because they share the same content. In this case, the authors propose using the 530 field with subfield code $\$$ a to indicate additional formats available. Although this subfield code is set as nonrepeatable when it is applied to general materials, the authors suggest setting it as repeatable to indicate all available alternative formats.

\section{4-Original Version Note}

The original version note field can be used to describe the original item that is the source of the alternative format material. However, existing records rarely use this field because they are created from the perspective of original materials using various fields. Thus current records may not use this field for original materials. The authors propose creating records on the basis of alternative format materials so that the 534 field is essential in describing the original materials. This field can indicate specific aspects of the materials using various subfield codes. In addition to the existing subfield codes, the authors suggest adding two new subfield codes $(\$ \mathrm{r}$ and $\$ s$ ) to identify the name and number of part/section of the original material if it is a part of sequencing form. Subfield code $\$ \mathrm{r}$ is added to describe the number of part/section and $\$ s$ is for the name of part/section of the original material.

\section{0-Added Entry-Personal Name}

The authors found many inconsistencies in use of the 700 field in the data set. Some records use this field to give access to the records of alternative format materials. Others use it only for the personal names related to original materials. To make clear how to use this field and to ensure consistency in describing alternative format materials, the authors propose setting some subfield codes as mandatory if the record is for an alternative format material:

- In case of sound recordings, the reader should be described with subfield code $\$$ e (relator term).

- In case of braille format, the braille translator or contributor (if an individual) should be described with subfield code $\$ \mathrm{e}$.

- Personal names for those who are responsible for (or related to) the content or publication of the alternative format material should be described in this field.

Using this field for the individuals related to an alternative format material can provide additional access points to the material.

\section{0-Added Entry-Corporate Name}

Some records occasionally use the 710 field for the corporate name related to alternative format materials, but others use it only for original materials. To guide consistency in using this field, the authors propose setting some subfield codes as mandatory if the record is for an alternative format material:

- In case of sound recordings, the institution that conducted the recording should be described with subfield code $\$$ e.

- In case of braille format, the institution that conducted the translation should be described with subfield code $\$$ e.

- Corporate names that are responsible for (or related to) the content of alternative-format materials should be described in this field.

As with the 700 field, the use of this field can provide additional access points to alternative format materials.

\section{6-Electronic Location and Access}

If an alternative format material is published in electronic format that can be accessed on the web, the electronic location and access field is used to indicate its URL or other detailed aspects of the materials.

\section{Addition of New Fields and Subfield Codes in KORMARC}

The existing fields in KORMARC, mostly adopted from MARC, are designed to describe general materials, especially monographs. Although these fields may be sufficient to describe monographs in detail and other types of materials to some degree, they may not be sufficient to describe alternative format materials that have unique characteristics. Therefore this paper proposes to add fields specific to alternative format materials.

\section{Additional Equipment Required to Use Alternative Format Materials}

With the development of information technology, alternative format materials have been published in various formats other than braille books. These new types of materials often require additional equipment to use their content. Therefore providing information about the equipment needed along with describing the material itself is desirable when creating records for these materials. This information can be described in the 500 field in plain text. However, assigning a specific field to precisely describe detailed aspects of the equipment is better.

A new field, 539-Equipment for Alternative Format Material, is proposed for this purpose. This field would include a general description of the equipment needed for specific types of alternative format materials, restrictions, 
and detailed characteristics of the equipment. The following subfield codes are to be placed with this field:

- \$a name of equipment [repeatable]

- \$b target audience [repeatable]

- $\$$ c restrictions on use [repeatable]

- $\$ g$ other information related to equipment [repeatable]

- $\$$ h target materials for the equipment [repeatable]

- \$u location of equipment [nonrepeatable]

Below is an example of the use of this new field:

539 \#\# \$a Screen reader; \$b Visually impaired; \$c Availability restricted to those meeting the eligibility criteria of the holding agency; $\$ g$ Use with Digital Pin Displayer; \$h Large print; \$u Disability resource center

Personal/Corporate Name Related to the Transformation of Alternative Format Materials

In current records for alternative format materials, the 500 and 511 fields are used to describe the braille translator and reader, respectively. However, the 500 field is designed to provide general information about the material that may not be properly described using other fields. Thus it covers a wide range of description related to those materials and puts the values in plain-text format. However, description about the braille translator may not be considered as general information and need to be described using separated fields.

A new field, 509-Alternative Format Translator/Contributor note, is proposed. Although this field is proposed primarily to describe the translators or contributor of alternative format materials, it also can be used for reader or other participant (institutions) in transforming those formats. The authors recommend using this field to describe the reader of the alternative format materials instead of using the 511 field because the reader in such materials is conceptually different from performer, participants, players, or presenters.

The subfield codes to be included in this field are the following:

- \$a personal name / corporate name [repeatable]

- $\$$ b name of department or institution where the person is affiliated [repeatable]

- \$e role [repeatable]

This example uses the 509 filed and an added entry field:

509 \#\# \$a Robert Molnar; \$b National Library of Korea; \$e Narration
700 1\# \$a Molnar, Robert; \$e Narration; \$u National Library of Korea

Some sound-recording materials are currently digitally transformed without any reader. In this case, the 516 field would be used to describe the publishing method. The person or institution in the 509 field also might be described in added entries in the 700 or 710 field.

\section{Type of Computer File or Data}

The 516 field is designed to describe the types of computer file or data and provides space for computer- or digitalrelated aspects of general materials. In the case of alternative format materials in digital format, this field is often used to indicate the types of file or data used in publishing those materials. However, the 516 field has only one subfield code, $\$ a$-type of computer file or data note, which is insufficient to fully describe the digital aspects of alternative format materials. To address this, the authors suggest expanding the subfield codes by adding $\$ \mathrm{~b}, \$ \mathrm{c}, \$ \mathrm{~d}$ and $\$ \mathrm{e}$. The expanded subfield codes in the 516 field are the following:

- \$a-type of computer file or data note [nonrepeatable]

- $\$ b$ - data type (alternative format material) [nonrepeatable]

- $\$ \mathrm{c}$ - computer file type (alternative format material) [nonrepeatable]

- $\$ \mathrm{~d}$-restrictions on running file or data [repeatable]

- $\$ \mathrm{e}$-other considerations on running file or data [repeatable]

Below is an example showing use of the 516 field:

516 \#\# \$b DAISY; \$c TTS(Text-To-Speech); \$d DAISY player; \$e Transformed from analog to digital

By using these expanded subfield codes, alternative format material in digital format, such as sound recordings in DAISY format, can be described in this field instead of using a plain-text statement in the 500 field.

\section{Conclusion}

KCR4 is the cataloging standard of South Korea that is designed to be adopted by all libraries in the country, including public libraries, university libraries, school libraries, and libraries for people with disabilities. However, KCR4 lacks detailed and clear rules for describing and representing specific aspects of alternative format material. The description rules it does provide for those materials focus mainly on 
describing braille books. As a result, other types of alternative format materials cannot be sufficiently described.

KORMARC, based on the Korean Cataloging Rules, is used to generate bibliographic records for various types of materials, including alternative format materials. However, KORMARC also lacks sufficient fields to describe the unique characteristics of those materials. This problem results in inconsistencies and ambiguity in bibliographic records for those materials.

To solve the problems that KCR4 and KORMARC present in describing alternative format materials, the authors propose revision of general cataloging rules in KCR4, modification of existing fields and subfield codes in KORMARC, and addition of new fields and subfield codes in KORMARC.

First, the authors propose creating records from the perspective of alternative format materials and adding descriptions of original materials using other fields such as the 534 field. By applying this approach, alternative format materials can be fully described using various fields in KORMARC and provide additional information in a consistent way. In addition, this paper expanded the types of alternative format materials from two ("Braille materials" and "Sound recordings") to twenty-four types to precisely indicate the characteristics of those materials.

Second, existing fields and subfield codes in KORMARC are revised or modified to eliminate inconsistencies and provide a standardized approach to creating records for alternative format materials. This revision and modification covers thirteen fields with subfield codes in KORMARC that can be applied to alternative format materials.

Third, new fields or subfield codes are added to existing fields to complement the sparse alternative format-specific fields. These fields include the following:

- 539_Additional equipment for alternative format materials

- 509-Alternative Format Translator/Contributor note

- 516-Type of computer file or data

This addition of fields and subfield codes is expected to support standardized and more detailed descriptions of those materials.

Through these proposed revision, modification, and addition of fields and subfield codes in KORMARC, the unique characteristics of alternative format materials can be fully described. It also can improve the retrieval of alternative format materials based on the detailed and consistent resource description. By retrieving and providing appropriate alternative format materials, the specific needs of people with different types of disabilities can be sufficiently satisfied.

Although this paper was limited to KCR4 and KORMARC currently used in South Korea, the proposed revisions and modifications may be applied to other cataloging rules and standards to improve the lack of details when describing various alternative format materials.

\section{References}

(Titles are given in English followed by the Korean title in brackets for those titles published in Korean.)

1. Linda Lou Wiler and Eleanor Lomax, "The Americans with Disabilities Act Compliance and Academic Libraries in the Southeastern United States," Journal of Southern Academic \& Special Librarianship 2, no. 1 (2000), accessed March 9, 2012, http://southernlibrarianship.icaap.org/content/v02n01/ wiler_101.html.

2. Patricia P. Nelson, "Library Services for People with Disabilities: Results of a Survey," Bulletin of the Medical Library Association 84, no. 3 (1996): 397-401.

3. Birgitta Irvall and Gyda Skat Nielsen, Access to Libraries for Persons with Disabilities-Checklist, IFLA Professional Reports no. 89 (The Hague, Netherlands: IFLA, 2005), accessed May 13, 2012, www.ifla.org/VII/s9/nd1/iflapr-89e .pdf.

4. Korean Library Association, Korean Cataloging Rules [한국 목록규칙], 1st ed. (Seoul: Korean Library Association, 1964).

5. Korean Library Association, Korean Cataloging Rules [한국 목록규칙], 3rd ed. (Seoul: Korean Library Association, 1983).

6. Taewoo Nam, “A Study on Theory of Cataloging [목록법 이 론에 대한 연구]," Journal of the Korean Society for Information Management 14, no. 1 (1997): 223-54.

7. Korean Library Association, Korean Cataloging Rules [한국 목록규칙], 4th ed. (Seoul: Korean Library Association, 2003).

8. Hye-Kyoung Kim and Taewoo Nam, "Analysis of the Chapter 1 'General Rules for Description' in the Draft of KCR4 [KCR 4판 초안 제1장 ‘기술총칙'에 관한 분석]," in Proceedings of the 9th Conference of Korean Society for Information Management, 169-74 (Seoul, South Korea: Korean Society for Information Management, 2002).

9. Alicia Julia Todaro, "Library Services for People with Disabilities in Argentina," New Library World 106, no. 5/6 (2005): 253-68.

10. "Disabilities," World Health Organization, 2012, accessed February 24, 2012, www.who.int/topics/disabilities/en.

11. "Introduction to Print Disability," International Federation of Library Associations and Institutions, Libraries Serving Persons with Print Disabilities Section, 2011, accessed May 15, 2012, www.ifla.org/files/libraries-for-print-disabilities/presentations/Introducing\%20Print\%20Disability.ppt.

12. National Library of Korea, Library Services for People with Disabilities Manual [도서관 장애인서비스 매뉴얼] (Seoul: Korean Library Association, 2009).

13. South Korea, Act on Welfare of Persons with Disabilities, Article 2, January 1, 2005 [장애인복지법 시행령]. 
14. Barbara Nail-Chiwetalu, "Guidelines for Accessing Alternative Format Educational Materials," NSL Other Writings, March 1, 2000, accessed February 27, 2012, www.loc.gov/nls/ other/guidelines.html.

15. Birgitta Irvall and Gyda Skat Nielsen, Access to Libraries for Persons with Disabilities-Checklist, IFLA Professional Reports no. 89 (2005), accessed May 13, 2012, www.ifla.org/ VII/s9/nd1/iflapr-89e.pdf.

16. National Library of Korea, Statistics on Library Services for
People with Disabilities [장애인도서관서비스 통계 현황자 료] (Seoul: National Library of Korea, 2011): 40-50.

17. Anglo-American Cataloguing Rules, 2nd ed., 2002 rev., 2005 update (Chicago: American Library Association; Ottawa: Canadian Library Association; London: Chartered Institute of Library and Information Professionals, 2002), 1.1C1: 1-10.

18. Ibid., 1-11.

19. Korean Library Association, Korean Cataloging Rules [한국 목록규칙], 4th ed. 